"This is the peer reviewed version of the following article: Foulkes, N.S., Eckel-Mahan, K., Cermakian, N. (2020) Remembering Paolo: A tribute to Paolo Sassone-Corsi. Journal of Pineal Research, 69:e12692. NB: Invited obituary, which has been published in final form at 10.1111/jpi.12692. This article may be used for non-commercial purposes in accordance with Wiley Terms and Conditions for Use of Self-Archived Versions."

\title{
Remembering Paolo
}

A tribute to Paolo Sassone-Corsi

Nicholas S. Foulkes ${ }^{1}$, Kristin Eckel-Mahan², Nicolas Cermakian ${ }^{3, *}$

Affiliations:

${ }^{1}$ Institute of Biological and Chemical Systems, Karlsruhe Institute of Technology, Hermann-von-

Helmholtz Platz 1, 76344 Eggenstein-Leopoldshafen, Germany

${ }^{2}$ Institute of Molecular Medicine, University of Texas Health Science Center at Houston, Houston, TX

USA

${ }^{1}$ Douglas Research Centre, McGill University, Montreal, QC, Canada, H4H 1R3

*Correspondence: Nicolas Cermakian, 6875 LaSalle boulevard, Douglas Research Centre, McGill

University, Montreal, QC, Canada, H4H 1R3; 514-761-6131 ext. 4936; nicolas.cermakian@mcgill.ca 
In what has been a challenging year for all of us, recently we have tragically lost one of the most prominent leaders of the clock biology community. Paolo Sassone-Corsi passed away suddenly on July $22^{\text {nd }}, 2020$, aged 64, at his home in Laguna Beach, California. Paolo will be remembered as a trail blazer in revealing the transcriptional and epigenetic mechanisms involved in circadian clock function, and in clock-controlled physiological systems, from metabolism to cancer.

As is often the case in the field of biological rhythms, Paolo was not originally trained as a clock biologist. His $\mathrm{PhD}$ research project was based in the field of yeast mitochondrial genetics. However, it was his postdoctoral training in the prestigious team of Pierre Chambon at the Laboratoire de Génétique Moléculaire des Eucaryotes (LGME) in Strasbourg, France, that served as the foundation for his future studies involving clock mechanisms. Paolo was involved in pioneering work in which eukaryotic viruses were used to study the basic principles of transcriptional control. Following his postdoctoral work, Paolo moved to the Salk Institute in San Diego, California and Inder Verma's laboratory. There, he maintained his research interest in transcriptional control, now focusing on the $c$ fos and c-jun oncogenes. Part of this work led to the discovery that serum-induced c-fos gene expression was autoregulated by the c-Fos protein, work that was in many ways a prelude for Paolo's future focus on the circadian clock where autoregulatory feedback loops are of course fundamental. During his stay at the Salk, Paolo also developed his skills to network and collaborate highly effectively as a way for him to bridge and capitalize on diverse areas of expertise.

Upon his return to France, Paolo started his own lab at the LGME, which later became the Institut de Génétique et de Biologie Moléculaire et Cellulaire (IGBMC). There, he chose to bring a more physiological focus to his work. In collaboration with his wife and long-term collaborator, Emiliana Borrelli, he screened a pituitary gland cDNA library for cAMP-responsive transcription factors that were homologous to CREB. Paolo reasoned that given the key role for cAMP as a second messenger in 
pituitary function, cAMP responsive factors might play a privileged role in this gland. This was an enlightened decision, as most work on transcription factors at this time focused on revealing their mechanisms of action. This work led to the identification of CREM, a gene subjected to extensive alternative splicing and differentially expressed in many neuroendocrine organs. The expression pattern of CREM naturally fostered three main research axes in Paolo's lab's for the next 15 years, namely pineal biology (and clock function), steroidogenesis, and spermatogenesis.

Attempts to understand the regulation of CREM expression in the testis led Paolo to collaborate with the group of Paul Pévet, at the University of Strasbourg. Paul Pévet was studying the effect of day length on the control of spermatogenesis and neuroendocrine axis function. The resulting collaboration between the two labs revealed the contribution of FSH in controlling CREM expression. The close links with the Pévet group, in particular Jörg Stehle, were also fundamental for subsequently revealing that CREM was expressed with a strong circadian rhythm in the pineal gland, under adrenergic control. This work coincided with the discovery in Paolo's lab that rhythmic CREM expression in the pineal gland was driven by an alternative cAMP-inducible promoter, which directed expression of a truncated CREM repressor termed ICER (Inducible cAMP early repressor). This represented one of the first examples of a rhythmically-expressed transcription factor in the pineal. Furthermore, the impressive dynamic expression profile of ICER together with the observation that this factor could actually regulate its own expression, highlighted the important potential of this type of gene regulatory circuit in conferring rhythmic transcription. To appreciate the relevance of this work, it is important to keep in mind that this discovery was made at a time when the only known clock genes were per and tim in fruit flies and frq in the fungus Neurospora. No clock genes had yet been identified in vertebrates and the concept of the transcription-translation feedback loop was still in its infancy. 
Paolo was quick to realize the opportunity of applying his own expertise in transcription control mechanisms to the emerging field of molecular clock mechanisms. His clock-related work moved away from a focus on the CREM gene and reoriented progressively towards a more general clock interest. A first aspect of this was the establishment of zebrafish as a new vertebrate animal model to study circadian clock biology and function. This was done in collaboration with an IGBMC colleague Uwe Strähle, who used zebrafish to study development of the embryonic nervous system. Several zebrafish clock gene homologs (Clock, Bmal, Per and Cry) were isolated and shown to be rhythmically expressed in various peripheral tissues, supporting the emerging general view of a hierarchical clock organization in vertebrates. However, unexpectedly, it was also shown that in contrast to the situation in mammals, circadian photoreception occurred in zebrafish in most peripheral tissues and even in cultured zebrafish fibroblasts. Paolo's lab in turn harnessed the power of mouse genetics to unravel clock molecular mechanisms and how they were regulated by environmental and physiological cues. One key set of experiments involved developing a model of fibroblast-collagen implants to simulate a peripheral tissue, which revealed that peripheral clocks could be controlled by central clock-controlled humoral circadian cues.

Consistent with his strong commitment to networking and collaboration, Paolo actively encouraged his colleagues in the lab, working on different topics, to cooperate with each other and so build bridges between their projects. For example, Paolo realized the great potential of combining one project studying the signal transduction during photoentrainment of the SCN clock and another the regulation of histone modifications. This led to the identification of light-induced phosphorylation of histone H3 in the SCN, which was the very first report of epigenetic modifications in relation to circadian clock function. This was the trigger for what became one of the main topics of the next 2 decades of Paolo's research: the interplay between epigenetics and circadian clocks. 
A subsequent step in this clock-epigenetics line of research was the striking discovery that a core clock transcription factor, CLOCK, has a histone acetyltransferase activity. This was published at a time of big changes for the lab: Paolo moved his laboratory to the University of California, Irvine, in 2006, taking up the position of Chair of UCI's Department of Pharmacology, before later moving to the Department of Biological Chemistry. While accompanied by a small number of his colleagues from Strasbourg, he rapidly built a cohesive research unit, and began to explore a new interest of his, circadian metabolism. This emphasis wove its way through the majority of the next 14 years of work, in which from epigenetic modifications to energy balance and aging, he helped prove that rhythmic metabolites and metabolism govern so much of our biology and physiology. Early work during this era centered on the extent to which clock proteins could interact with histone-modifying enzymes, and the ramifications such interactions could have on cellular metabolism and gene expression, as exemplified by rhythmicity in nicotinamide adenine dinucleotide (NAD+) and NAD+-dependent sirtuin activity. Though initially focused on individual clock-interacting proteins, his work expanded to address the impact of dietary nutrients on tissue-specific clock function and metabolism more globally.

During this time, he worked ardently to found the Center for Epigenetics and Metabolism at UCI, which currently encompasses a large number of internal and external members devoted to the impact of metabolism on gene regulation and disease prevention. A product of this emphasis was the discovery that dietary intake controls circadian metabolism in a clock-dependent manner, and that tissue-specific clocks communicate with one another in ways that get disrupted under specific forms of nutrient stress. In a collaborative effort with Pierre Baldi at UCI, Paolo helped compile a large database called CircadiOmics, which to this day serves as the largest publicly available compilation of circadian cellular events in the metabolome, transcriptome, acetylome, and proteome, from a range of cells, tissues, and organisms under different environmental and genetic perturbations. This has been a valuable resource for the circadian and metabolism fields. During the last few years of his life, Paolo's 
vision and passion for science was perhaps best exemplified by several high-impact studies addressing the importance of tissue-specific clock function in disease and aging prevention. Research during these years led to the discovery that the liver clock responds strongly to non-localized tumor burden and that environmental measures (such as caloric restriction) that can delay aging may function in part through circadian changes in protein acetylation.

Paolo's passing shocked everyone who knew him, not only because it was unexpected, but also because he was such a lively person, full of contagious energy, and enthusiastic about so many things in science and beyond. In fact, it is likely that his character was one of the secrets of his success in his many collaborations. Anyone who has worked with Paolo at some point during the four and a half decades of his research career, cannot fail to have been impressed and influenced by his enthusiasm for life and research. As a researcher, he was a go-getter: always ready to dive into new areas, to tackle new questions. He instinctively knew what was the next "hot" question and he had a great ability to identify and cooperate with the best collaborators to enable him to build a particular story in that direction. Added to this, his profound optimism and capacity to see the positive side of things, allowed him to succeed in addressing new questions.

He will be remembered as a great mentor, with an impressive ability to guide those working with him to work hard and to do great science. Even when the data we presented might have unseated many, after a discussion with Paolo, we always left the office feeling that he had extracted the one positive idea out of what had seemed to us an absolute shipwreck. We will forever thank him for his unique vision, his hard work, and his unwavering optimism, that carried many of us trainees through our mentored phase. 
Paolo's scientific interests were not only limited to biology. Since his childhood, Paolo had a passion for astronomy, owned a telescope at home and was always excited about the next major astronomical event. Paolo was born in Naples and like so many Neapolitans, he loved football, great pizza and life by the sea (which was likely one of the triggers to eventually move back to California, with direct sight of the Pacific Ocean). Paolo came a long way from his childhood in the shadow of Vesuvius to his life as a leading clock biologist on the Pacific coast of California. However, clearly, his Southern Italian genes stayed with him all the way and participated in his unique personality.

We will miss him immensely, and his life and work will be a constant comfort to us. It is with honor that we will continue to pursue his goals to the best of our abilities.

Ciao Paolo, ci mancherai!

Note: The authors were all postdoctoral fellows in the lab of Paolo Sassone-Corsi at key stages in his career (NSF, 1989-2000; NC, 1998-2000; and KLM, 2008-2015). 